\title{
Gambogic acid prevents angiotensin II-induced abdominal aortic aneurysm through inflammatory and oxidative stress dependent targeting the PI3K/Akt/mTOR and NF-kB signaling pathways
}

\author{
QIANG LIU ${ }^{1}$, PENG SHAN ${ }^{2}$ and HAIBIN LI ${ }^{1}$ \\ ${ }^{1}$ Department of Vascular Surgery, The First Hospital of Qiqihar City, Qiqihar, Heilongjiang 161021; \\ ${ }^{2}$ Department of Vascular Surgery, The First Affiliated Hospital of Harbin Medical University, \\ Harbin, Heilongjiang 150036, P.R. China
}

Received October 8, 2016; Accepted September 12, 2017

DOI: $10.3892 / \mathrm{mmr} .2018 .9720$

\begin{abstract}
Gamboge is the dry resin secreted by Garcinia hanbaryi Hook.f, with the function of promoting blood circulation and anti-cancer effects, detoxification, hemostasis and killing insects. It is also used for the treatment of cancer, brain edema and other diseases. Gambogic acid is the main effective constituent of Gamboge. The present study tested the hypothesis that the effect of Gambogic acid prevents angiotensin II-induced abdominal aortic aneurysm (AAA), and explored its underlying mechanism. It was demonstrated that gambogic acid significantly inhibited AAA incidence rate, and reduced edge leading aortic diameter and aortic wall thickness in AAA mice. Gambogic acid treatment markedly decreased the levels of proinflammatory cytokines and oxidative stress factors, and transforming growth factor- $\beta$ (TGF- $\beta$ ) and matrix metalloproteinase (MMP)-2 and MMP-9 protein expression in AAA mice. Furthermore, Gambogic acid decreased expression of phosphatidylinositol 3-kinase (PI3K), and phosphorylation of protein kinase B (Akt), mechanistic target of rapamycin (mTOR) and p70-S6 kinase 1. It also suppressed nuclear factor (NF)- $\mathrm{kB}$ protein expression in AAA mice. The findings of the present study indicated that Gambogic acid prevents angiotensin II-induced AAA through inflammatory and oxidative stress-dependent targeting of the $\mathrm{PI} 3 \mathrm{~K} / \mathrm{Akt} / \mathrm{mTOR}$ and NF-kB signaling pathways.
\end{abstract}

\section{Introduction}

Aneurysm is caused by injured normal structure of the aortic wall due to congenital or acquired disorders, especially by

Correspondence to: Dr Peng Shan, Department of Vascular Surgery, The First Affiliated Hospital of Harbin Medical University, 23 Youzheng Street, Harbin, Heilongjiang 150036, P.R. China E-mail: wfexdqkt98@126.com

Key words: gambogic acid, abdominal aortic aneurysm, phosphatidylinositol 3-kinase/protein kinase $\mathrm{B} /$ mechanistic target of rapamycin, nuclear factor- $\mathrm{kB}$ an injured elastic fiber layer of arterial walls, which leads to the gradual dilation or enlargement of the aorta in local or multiple places (1). In general, aneurysm refers to an artery with a diameter exceeding $50 \%$ of the normal size. Abdominal aortic aneurysm (AAA) refers to a high-risk disease caused by the rupture of blood vessel walls under the impact of blood flow, due to abnormal dilation or limited expansion of abdominal aorta (1). The incidence rate of AAA in China has reached $2 \%$, and increases year by year (2). Due to the lack of understanding towards the incidence and underlying molecular mechanisms of AAA, in addition to surgery, there is no drug which can effectively treat or slow down the development of AAA (3).

According to previous studies, AAA may be associated with smoking, sex, oxidative stress, matrix protein, blood lipid levels and other factors (4). The activities of reactive oxygen species, $\mathrm{O}_{2}$, nicotinamide adenine dinucleotide phosphate (NADPH) oxidase, and the expression of NADPH oxidase p47phox ligand, were demonstrated to be increased in human AAA tissue samples (5). In addition, matrix metalloproteinase (MMP) was also detected in human AAA tissue samples; increased activity of MMPs promotes the degradation of elastin and collagen in artery walls, leading to the expansion of arterial walls (6). Moreover, AAA destroys the extracellular matrix, all of which results in AAA (7). Studies also demonstrated that triglycerides and cholesterol are also associated with the incidence of AAA $(6,7)$.

Phosphatidylinositol 3-kinase (PI3K), a member of the kinase family, is widely distributed in the human body. In the 1980s, the PI3K family attracted the attention of the medical field (8). PI3K, an important catalytic enzyme system regulating metabolic pathways of phospholipids, is produced by phospholipid messenger molecules. It specifically binds to the phosphatidylinositol base and transfers the phosphate group of ATP to phosphorylate PI into inositol lipids (8). Lymphokine-activated killer T-cell-originated protein kinase (PBK) forms an important signaling pathway, termed the PI3K-protein kinase B (Akt) signaling pathway, which together with Akt, located in its downstream, serves an important role in the survival, differentiation, proliferation and apoptosis of cells (9). 
Nuclear factor (NF)- $\kappa \mathrm{B}$, a transcription factor, has been confirmed to be widely distributed in eukaryotic cells, and can quickly transfer into the nucleus and specifically bind to special sites of cell gene promoters or enhancer sequences, to promote transcription and expression (10). Studies have confirmed that NF- $\mathrm{NB}$ is closely associated with many major pathophysiological processes, such as the immune response and inflammation as well as the proliferation, differentiation, metastasis and apoptosis of tissue cells, serving an important role in the incidence and development of many diseases $(11,12)$.

Gamboge is the dry resin secreted by Garcinia hanbaryi Hook.f. Gamboge mainly consists of $70-80 \%$ resin and $15-25 \%$ gum (13). The major constituents of gamboge include gambogic acid, neogambogic acid, allogambogic acid, morellin, isomorellin, morellic acid and isomorellic acid, among which gambogic acid is the main effective constituent (14). In recent years, many studies have further investigated the antineoplastic mechanism of gambogic acid, proving that gambogic acid can induce cell differentiation and tumor cell apoptosis, as well as inhibiting angiogenesis and lowering the activity of telomerase to block the cell cycle and reverse drug-resistance, so as to kill tumor cells (15). The present study aimed to test the effect of gambogic acid on the prevention of angiotensin (Ang) II-induced AAA, and to explore its underlying mechanism.

\section{Materials and methods}

Animals. Animal experiments were performed according to protocols approved by the institutional animal care and use committee of The First Hospital of Qiqihar City. The present study was approved by the Animal Ethical and Welfare Committee of The First Hospital of Qiqihar City (Qiqihar, China). Male C57BL/6 mice (ApoE ${ }^{-/-}$mice, 20-22 g, 6 weeks old; $n=46$ ) were purchased from Animal Laboratory of Harbin Medical Sciences University (Harbin, China) and were raised in specific pathogen-free conditions with a 12-h light/dark cycle at $24 \pm 2^{\circ} \mathrm{C}$ and $50-60 \%$ humidity. An AAA model was induced by chronic infusion of $1,000 \mathrm{ng} / \mathrm{kg} / \mathrm{min}$ Ang II using mini-osmotic pumps. All mice were randomly distributed into five groups: Sham ( $\mathrm{n}=6)$, AAA model ( $\mathrm{n}=10), 5 \mathrm{mg} / \mathrm{kg}$ Gambogic acid $(\mathrm{n}=10)$, $10 \mathrm{mg} / \mathrm{kg}$ Gambogic acid $(\mathrm{n}=10)$ and $20 \mathrm{mg} / \mathrm{kg}$ Gambogic acid $(n=10)$. Sham and AAA model groups were gavaged with normal saline. The 5,10 and $20 \mathrm{mg} / \mathrm{kg}$ Gambogic acid groups were gavaged with 5,10 or $20 \mathrm{mg} / \mathrm{kg}$ every 3 days Gambogic acid, respectively, for 4 weeks.

Staining of toluidine blue. All mice were anesthetized with sodium pentobarbital $(50 \mathrm{mg} / \mathrm{kg}$; Sigma-Aldrich; Merck KGaA, Darmstadt, Germany) and sacrificed. The aortas of all mice were immediately separated, washed in PBS, and perfused with $4 \%$ paraformaldehyde for $30 \mathrm{~min}$. Tissue samples were embedded into paraffin and cut into 5-6 $\mu \mathrm{M}$ sections. All tissue sections were deparaffinized and hydrated in several changes of ethanol and Tissue-Clear ${ }^{\circledR}$ (Sakura Finetek Europe B.V., Flemingweg, The Netherlands). All tissue sections were stained with toluidine blue working solution at room temperature for $30 \mathrm{~min}$ and dehydrated with ethanol. Samples were normalized to aortic vessel wall area $\left(\mathrm{mm}^{2}\right)$ and total numbers per aorta since were calculated in numbers.
ELISA kits. All mice were anesthetized with sodium pentobarbital (50 mg/kg) and sacrificed. Aortic tissues samples (50 mg) were homogenized with radioimmunoprecipitation assay (RIPA) lysis buffer (Beyotime Institute of Biotechnology, Haimen, China) on ice for $15 \mathrm{~min}$ and centrifuged at $14,000 \times \mathrm{g}$ at $4^{\circ} \mathrm{C}$ for $10 \mathrm{~min}$. Protein content was measured by Bicinchoninic Acid (BCA) assay (Beyotime Institute of Biotechnology), and equal protein $(10 \mu \mathrm{g})$ was incubated with corresponding ELISA kits. Tumor necrosis factor- $\alpha$ (TNF- $\alpha$; cat. no. H052), interleukin (IL)-1 $\beta$ (cat. no. H052), IL-6 (cat. no. H002), IL-18 (cat. no. H015), glutathione peroxidase (GSH-PX; cat. no. A005), GSH (cat. no. A006-2), malondialdehyde (MDA; cat. no. A003-1) and superoxide dismutase (SOD; cat. no. A001-1-1) levels were measured using ELISA kits (Nanjing Jiancheng Biological Engineering Institute, Nanjing, China). The absorbance was measured in a spectrophotometer (Bio-Rad Laboratories, Inc., Hercules, CA, USA) at $450 \mathrm{~nm}$.

Western blot analysis. All mice were anesthetized with sodium pentobarbital $(50 \mathrm{mg} / \mathrm{kg})$ and sacrificed. Aortic tissues samples $(50 \mathrm{mg})$ were homogenized with RIPA lysis buffer on ice for $15 \mathrm{~min}$ and centrifuged at $14,000 \mathrm{x} \mathrm{g}$ at $4^{\circ} \mathrm{C}$ for $10 \mathrm{~min}$. Protein content was measured using BCA assay. Equal protein $(50 \mu \mathrm{g})$ was separated on $10 \%$ SDS-PAGE gels and blotted onto a nitrocellulose membrane (EMD Millipore, Billerica, MA, USA). The membrane was blocked with $5 \%$ non-fat powdered milk in TBS with Tween-20 for $1 \mathrm{~h}$ at $37^{\circ} \mathrm{C}$, and incubated with the following primary antibodies: transforming growth factor (TGF)- $\beta$ (cat. no. sc-7892; 1:500), MMP-2 (cat. no. sc-10736; 1:500), MMP-9 (cat.no. sc-10737; 1:500), PI3K (cat. no. sc-7174; 1:500), phosphorylated (p)-Akt (cat. no. sc-7985-R; 1:500), p-mechanistic target of rapamycin (mTOR; cat. no. sc-101738; 1:500; all Santa Cruz Biotechnology, Inc., Dallas, TX, USA), p-p70-S6 kinase 1 (cat. no. p-p70S6K1; 9204; 1:2,000; Cell Signaling Technology, Inc.), NF-кB (cat. no. sc-109; 1:500) and GAPDH (cat. no. sc-25778; 1:500; both Santa Cruz Biotechnology, Inc.) overnight at $4^{\circ} \mathrm{C}$, followed by incubation with horseradish peroxidase-conjugated secondary antibodies (cat. no. sc-2030; 1:5,000; Santa Cruz Biotechnology, Inc.) at $37^{\circ} \mathrm{C}$ for $1 \mathrm{~h}$. Membrane was developed using Enhanced Chemiluminescence Prime Western Blotting reagent (GE Healthcare Life Sciences, Little Chalfont, UK) and calculated using GeneTools software using a Syngene gel documentation system.

Statistical analysis. Data are expressed as the mean \pm standard deviation using SPSS 19.0 (IBM, Corp. Armonk, NY, USA). Data were analyzed using one-way analysis of variance followed by Dunnett's post hoc test. $\mathrm{P}<0.05$ was considered to indicate a statistically significant difference.

\section{Results}

Effect of Gambogic acid on AAA incidence rate in AAA mice. The chemical structure of Gambogic acid is presented in Fig. 1. As demonstrated in Fig. 2, the AAA incidence rate of AAA model group was significantly higher than that of sham group. Treatment with 5 and $10 \mathrm{mg} / \mathrm{kg}$ Gambogic acid significantly inhibited AAA incidence rate of AAA mice, compared with the AAA model group (Fig. 2). 


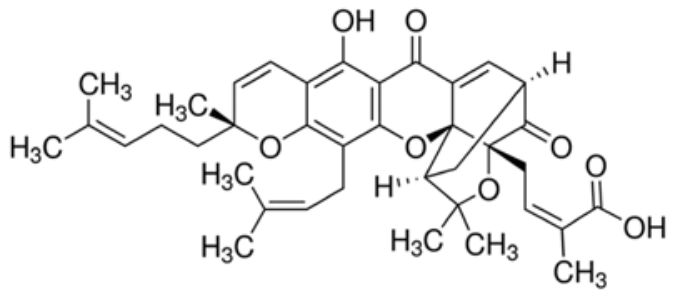

Figure 1. Chemical structure of Gambogic acid.

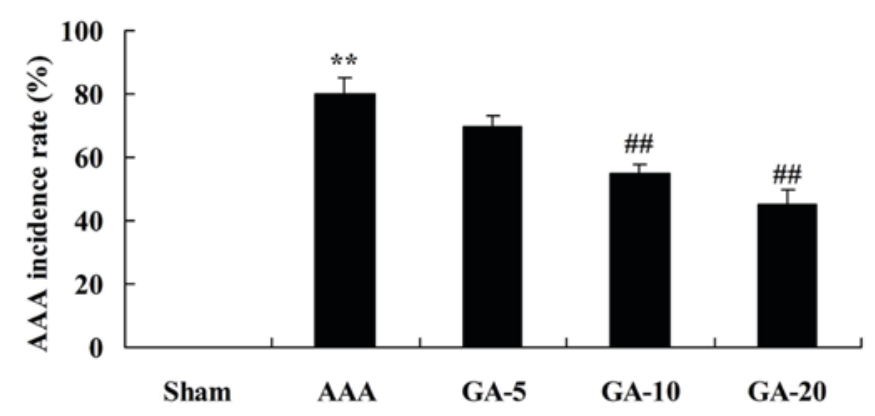

Figure 2. Effect of Gambogic acid on AAA incidence rate in AAA mice. Data are expressed as the mean \pm standard deviation. ${ }^{* *} \mathrm{P}<0.01$ vs. sham group; ${ }^{\# \#} \mathrm{P}<0.01$ vs. AAA model group. Sham, sham group; AAA, AAA model group; GA-5, Gambogic acid treatment group (5 mg/kg); GA-10, Gambogic acid treatment group $(10 \mathrm{mg} / \mathrm{kg}) ; \mathrm{GA}-20$, Gambogic acid treatment group $(20 \mathrm{mg} / \mathrm{kg})$; AAA, abdominal aortic aneurysm.

Effect of Gambogic acid on vascular remodeling in AAA mice. As presented in Fig. 3, there was a significant increase of edge leading aortic diameter and aortic wall thickness in AAA model mice, compared with the sham control group. Furthermore, 5 and $10 \mathrm{mg} / \mathrm{kg}$ Gambogic acid significantly reduced edge leading aortic diameter and aortic wall thickness in AAA mice, compared with the AAA model group (Fig. 3).

Effect of Gambogic acid on inflammation reactions in $A A A$ mice. As demonstrated in Fig. 4, TNF- $\alpha$, IL-1 $\beta$, IL-6 and IL-18 contents of AAA model mice were markedly higher than those of the sham control group. However, 5 and $10 \mathrm{mg} / \mathrm{kg}$ Gambogic acid treatment significantly decreased TNF- $\alpha$, IL-1 $\beta$, IL- 6 and IL-18 contents in AAA mice, compared with the AAA model group (Fig. 4).

Effect of Gambogic acid on oxidative stress in AAA mice. As presented in Fig. 5, GSH-PX, GSH and SOD levels were significantly inhibited, and MDA levels were significantly promoted, in the AAA model group, compared with the sham control group. Gambogic acid (5 and $10 \mathrm{mg} / \mathrm{kg}$ ) significantly increased GSH-PX, GSH and SOD levels, and reduced MDA levels in AAA mice, compared with the AAA model group (Fig. 5).

Effect of Gambogic acid on TGF- $\beta, M M P-2, M M P-9$ and $N F-\kappa B$ protein expression in $A A A$ mice. Western blotting demonstrated that TGF- $\beta$, MMP-2, MMP-9 and NF- $\kappa$ B protein expression in AAA model mice was significantly increased, compared with the sham control group (Fig. 6). However, compared with the AAA model groups, 5 and $10 \mathrm{mg} / \mathrm{kg}$ Gambogic acid treatment significantly suppressed
TGF- $\beta$, MMP-2, MMP-9 and NF- $\kappa$ B protein expression in AAA mice (Fig. 6).

Effect of Gambogic acid on PI3K, p-Akt, p-mTOR and $p$-p70S6K1 protein expression in AAA mice. Western blotting was performed to elucidate the potential role of PI3K, p-Akt, p-mTOR and p-p70S6K1 in AAA mice treated by Gambogic acid. As presented in Fig. 7, PI3K, p-Akt, p-mTOR and p-p70S6K1 protein expression were significantly increased in AAA mice compared with the sham control group. Gambogic acid treatment significantly induced PI3K, p-Akt, p-mTOR and p-p70S6K1 protein expression in AAA mice, compared with the AAA model group (Fig. 7).

\section{Discussion}

AAA is one of the most dangerous vascular degenerative diseases in vascular surgery. In the most serious cases, as the weak artery walls cannot withstand the impact of blood flow, AAA will lead to the rupture of walls of aneurysm, causing sudden mortality (16). With the aging population of China, the incidence of AAA is increasing year by year, and has become one of the diseases that threatens the life and health of many people (17). With the deepening of the understanding towards AAA and the development of imaging examination approaches, the diagnosis rate of the disease has been greatly improved, but its specific pathogenesis remains unknown (18). At present, people have demonstrated that the incidence of AAA is associated smoking, sex, oxidative stress, matrix proteins and blood lipids (19). The latest research has revealed that the inflammatory reaction serves an important role in the incidence and development of AAA (20). In this study, it was demonstrated that gambogic acid significantly inhibited the rate of AAA incidence, and reduced edge leading aortic diameter and aortic wall thickness in AAA mice. The results demonstrated that gambogic acid had an obvious improving effect on AAA.

In addition to the above factors, the incidence of AAA is also closely associated with the inflammatory reaction (21). According to current research, AAA is a chronic inflammatory disease, which is characterized by continuous arterial dilatation mainly caused by the invasion of inflammatory cells and the destruction of intermediate elastic protein matrix (22). Numerous inflammation-associated factors are closely associated with the pathogenesis of AAA (22). In the present study, it was demonstrated that Gambogic acid treatment significantly decreased TNF- $\alpha$, IL-1 $\beta$, IL- 6 and IL-18 contents, increased GSH-PX, GSH and SOD levels, and reduced MDA levels in AAA mice. Wen et al (23) suggested that Gambogic acid exhibits anti-psoriatic efficacy through inhibition inflammation.

In the case of the TGF- $\beta$ neutralizing antibody, the T lymphocyte deletion signal transducer as well as signal transducer and activator of transcription 3 also significantly promote the incidence of AAA induced by AngII (24). In a previous study, when AngII was used to induce AAA in C57 or low density lipoprotein receptor-deficient mice, the activity of TGF- $\beta$ was inhibited, leading to the necrosis of smooth muscular cells, degradation of elastin, exacerbation of intravascular inflammation in mice, thus eventually worsening AAA (24). Similarly, as a calcineurin immune-suppressing drug, cyclosporin-A can 

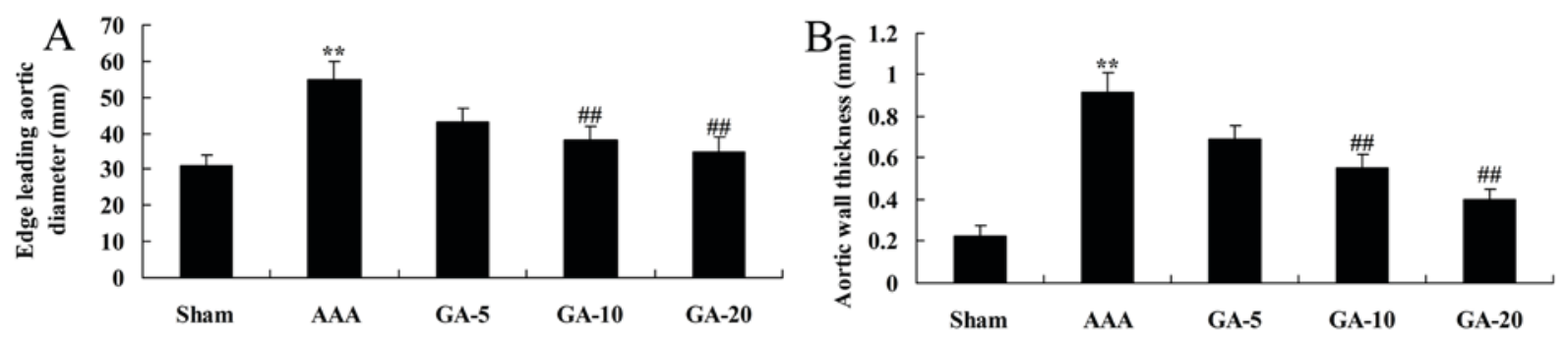

Figure 3. Effect of Gambogic acid on vascular remodeling in AAA mice. The effect of Gambogic acid on (A) edge leading aortic diameter and (B) aortic wall thickness in AAA mice. Data are expressed as the mean \pm standard deviation. ${ }^{* *} \mathrm{P}<0.01$ vs. sham group; ${ }^{\# \#} \mathrm{P}<0.01$ vs. AAA model group. Sham, sham group; AAA, AAA model group; GA-5, Gambogic acid treatment group (5 mg/kg); GA-10, Gambogic acid treatment group (10 mg/kg); GA-20, Gambogic acid treatment group $(20 \mathrm{mg} / \mathrm{kg})$; AAA, abdominal aortic aneurysm.
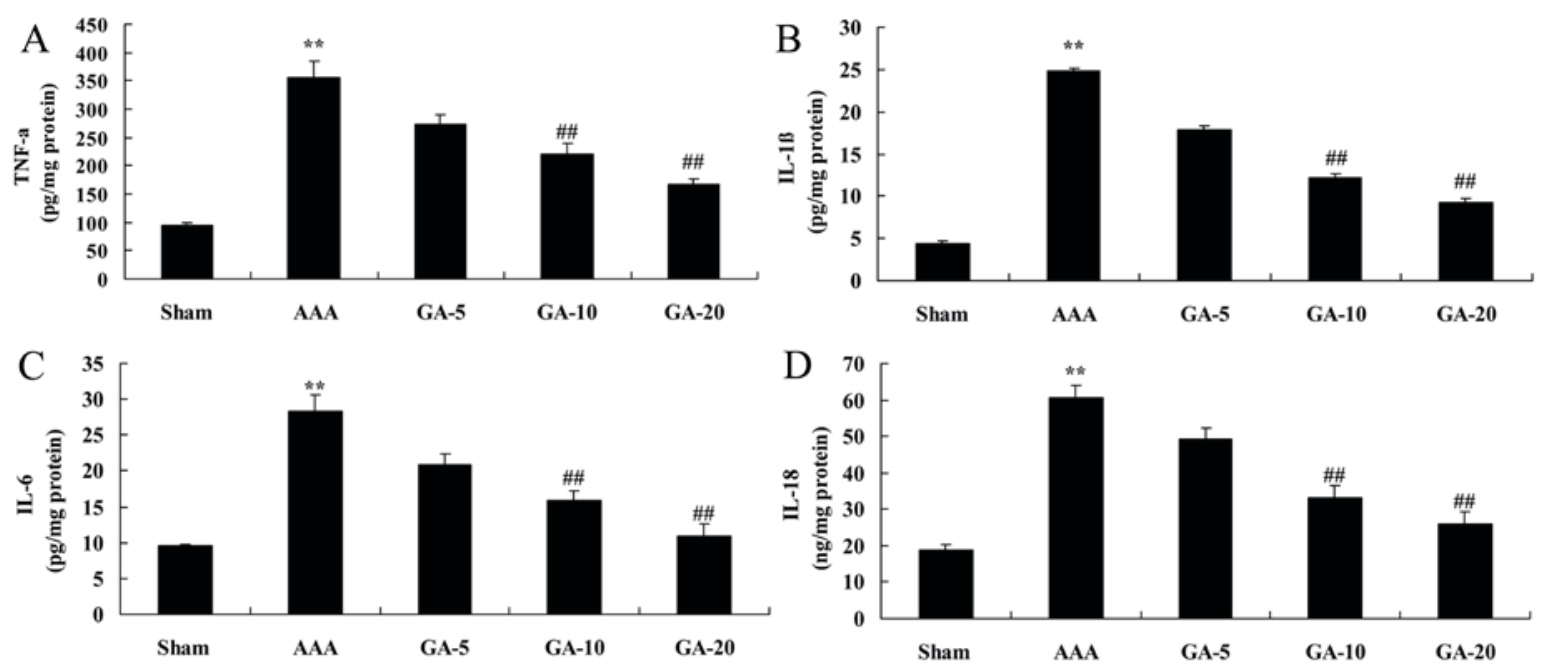

Figure 4. Effect of Gambogic acid on inflammation reactions in AAA mice. The effect of Gambogic acid on (A) TNF- $\alpha$, (B) IL-1 $\beta$, (C) IL-6 and (D) IL-18 levels in AAA mice. Data are expressed as the mean \pm standard deviation. ${ }^{* *} \mathrm{P}<0.01$ vs. sham group; ${ }^{\# \#} \mathrm{P}<0.01$ vs. AAA model group. Sham, sham group; AAA, AAA model group; GA-5, Gambogic acid treatment group (5 mg/kg); GA-10, Gambogic acid treatment group (10 mg/kg); GA-20, Gambogic acid treatment group $(20 \mathrm{mg} / \mathrm{kg}) ;$ AAA, abdominal aortic aneurysm; TNF- $\alpha$, tumor necrosis factor- $\alpha$; IL, interleukin.

promote the transcription of TGF- $\beta 1$ and activate latent TGF- $\beta 1$, thereby alleviating AAA induced by elastase or calcium chloride infusion; on the contrary, the TGF- $\beta$ antibody offsets the effect of cyclosporin-A in the treatment of AAA, suggesting that TGF- $\beta$ serves an important role in the incidence and development of AAA (25). In the present study, the results suggested that Gambogic acid treatment significantly suppressed TGF- $\beta$ protein expression in AAA mice. Qu et al (26) observed that Gambogic acid prevented pulmonary fibrosis by suppressing the TGF- $\beta 1 /$ Smad3 signaling pathway.

MMP is the major protease that causes the degradation of extracellular matrix of arterial walls. Matrix metalloproteinases are a series of homologous zinc- and calcium-dependent proteases, which exist in the form of inactive zymogen (27). The extracellular matrix, including elastic fibers, collagen, laminin and fibronectin, can be degraded through the cutting and activation of $\mathrm{N} 2$ end by enzyme, and has been recognized to serve a very important role in the incidence and development of aortic aneurysm (28). Most members of the MMP family serve important role in this process, in which MMP-2 and MMP-9 are especially important (29). Studies on arterial medial smooth muscle cells have demonstrated that normal arterial smooth muscle cells are the main components of artery intima media, which are not only associated with the diastolic and systolic function of arterial walls, but also regulate the synthesis and repair of extracellular matrix components, such as elastins and collagens $(27,29)$. They also demonstrated that Gambogic acid significantly inhibits MMP-2 and MMP-9 protein expression in AAA mice. Qi et al (30) indicated that Gambogic acid induced suppression of MDA-MB-435 human breast carcinoma cell lung metastasis through mediation of MMP-2/9 expression inhibition.

Regarding the association between PI3K and tumors, disorder of the PI3K-Akt signaling pathway has been demonstrated to lead to a variety of human cancers, including lung cancer, nasopharyngeal cancer, liver cancer, gastrointestinal cancer, breast cancer, ovarian cancer, renal cancer, prostate cancer, lymphoma, malignant glioma and medulloblastoma (31). The association between PI3K and non-tumor diseases, such as liver fibrosis, Alzheimer's disease, diabetes and cardiovascular disease has also attracted significant attention. It was demonstrated that selectively inhibiting the PI3K-Akt signaling pathway can promote the autophagy of macrophages, reduce the infiltration of plaque macrophages, and significantly alleviate the inflammatory response, thus improving atherosclerotic plaque (32). PI3K also contributes to the progression of atherosclerosis 

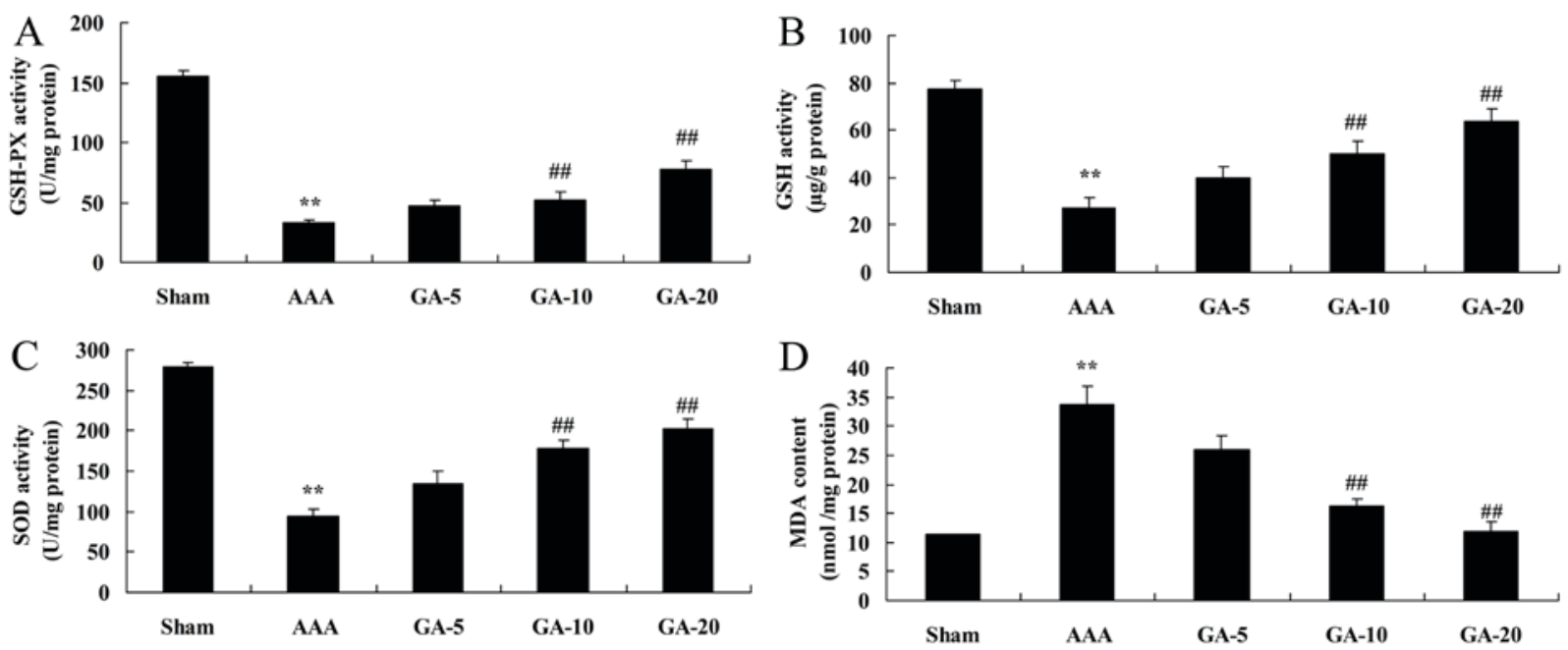

Figure 5. Effect of Gambogic acid on oxidative stress in AAA mice. The effect of Gambogic acid on (A) GSH-PX, (B) GSH, (C) SOD and (D) MDA in AAA mice. Data are expressed as the mean \pm standard deviation. ${ }^{* *} \mathrm{P}<0.01$ vs. sham group; ${ }^{\# \#} \mathrm{P}<0.01$ vs. AAA model group. Sham, sham group; AAA, AAA model group; GA-5, Gambogic acid treatment group $(5 \mathrm{mg} / \mathrm{kg})$; GA-10, Gambogic acid treatment group (10 mg/kg); GA-20, Gambogic acid treatment group (20 mg/kg); AAA, abdominal aortic aneurysm; GSH, glutathione; GSH-PX, glutathione peroxidase; SOD, superoxide dismutase; MDA, malondialdehyde.
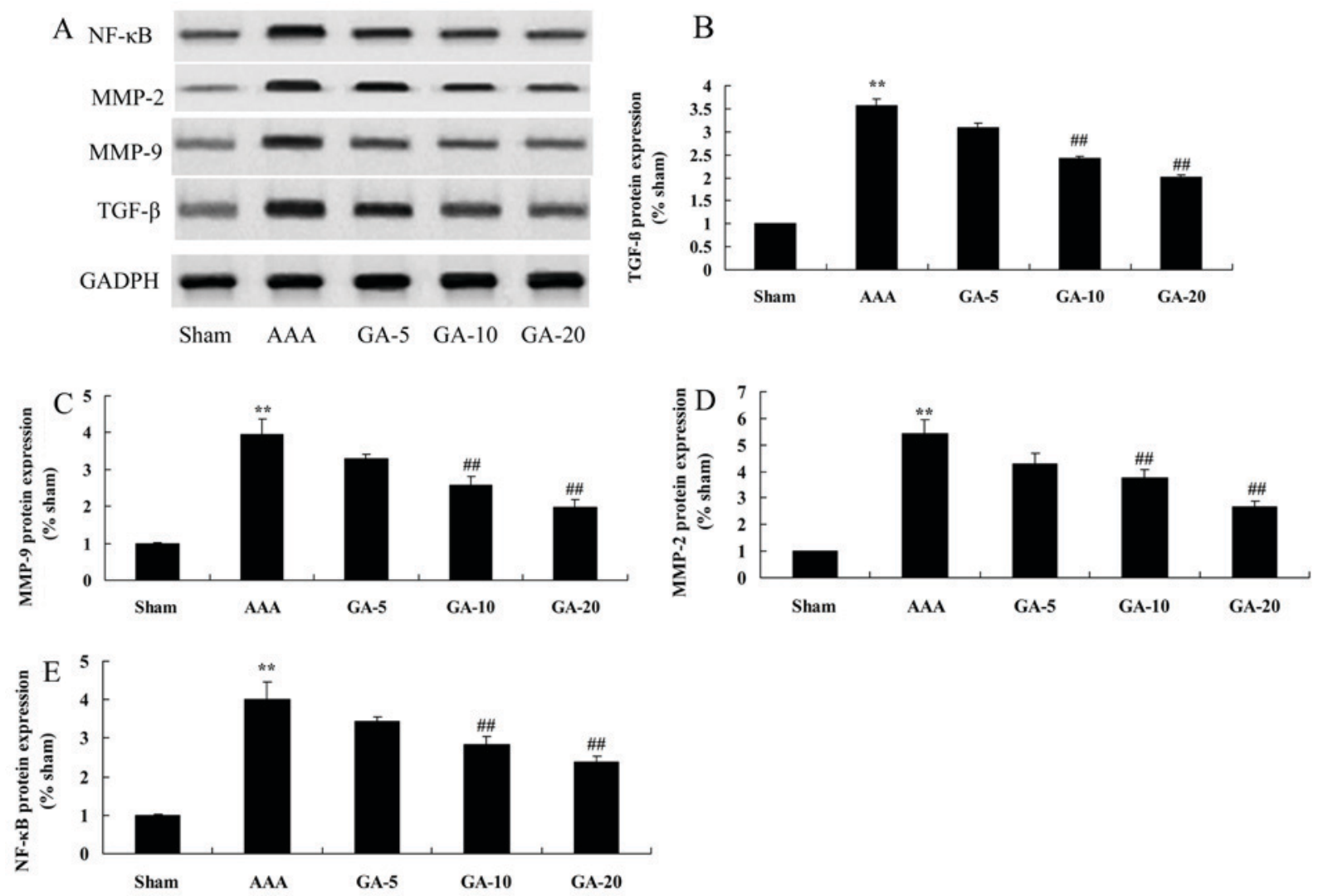

Figure 6. Effect of Gambogic acid on TGF- $\beta$, MMP-2, MMP-9 and NF- $\mathrm{kB}$ protein expression in AAA mice. Data are expressed as the mean \pm standard deviation. ${ }^{* *} \mathrm{P}<0.01$ vs. sham group; ${ }^{\# \#} \mathrm{P}<0.01$ vs. AAA model group. (A) Representative western blot images. Quantification of (B) TGF- $\beta$, (C) MMP-9, (D) MMP-2 and (E) NF-кB protein expression levels. Sham, sham group; AAA, AAA model group; GA-5, Gambogic acid treatment group (5 mg/kg); GA-10, Gambogic acid treatment group $(10 \mathrm{mg} / \mathrm{kg})$; GA-20, Gambogic acid treatment group $(20 \mathrm{mg} / \mathrm{kg})$; AAA, abdominal aortic aneurysm; TGF- $\beta$, transforming growth

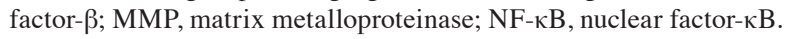

through affecting vascular endothelial cells, using a variety of signals can be transduced and regulated through the PI3K-Akt signaling pathway. Regulation of this pathway can directly or indirectly promote the pathological progression of atherosclerosis (9). In the present study, it was observed that Gambogic acid treatment significantly induced PI3K, p-Akt, p-mTOR and p-p70S6K1 protein expression in AAA mice. Wang et al identified that Gambogic acid suppresses the activity of multiple myeloma cells through the PI3K-Akt signaling pathway (33). 
A
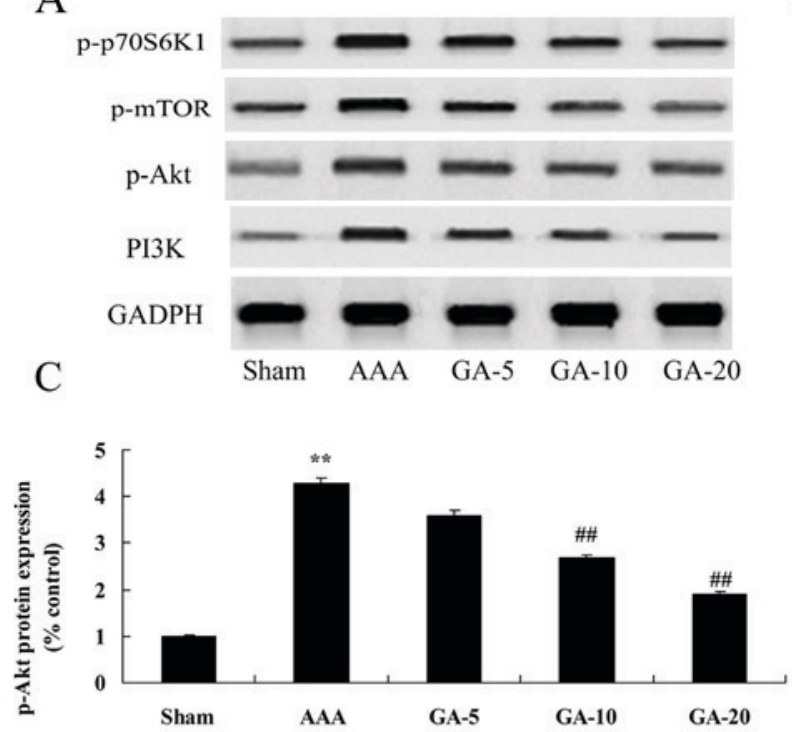

B

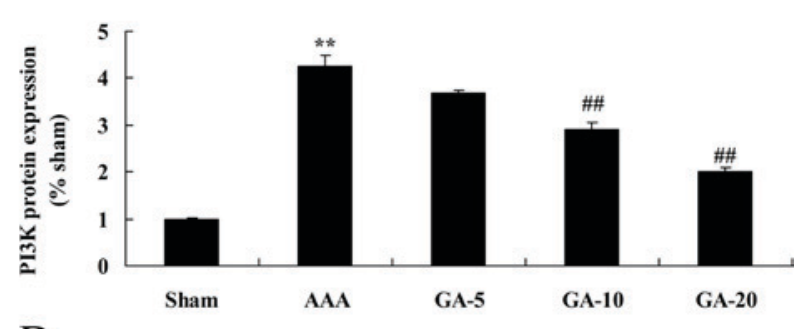

$\mathrm{D}$

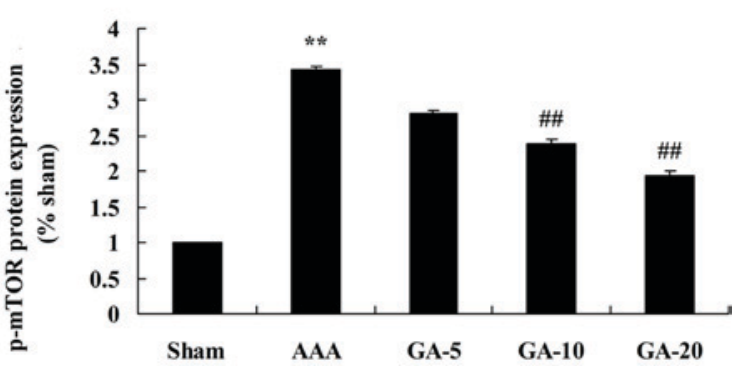

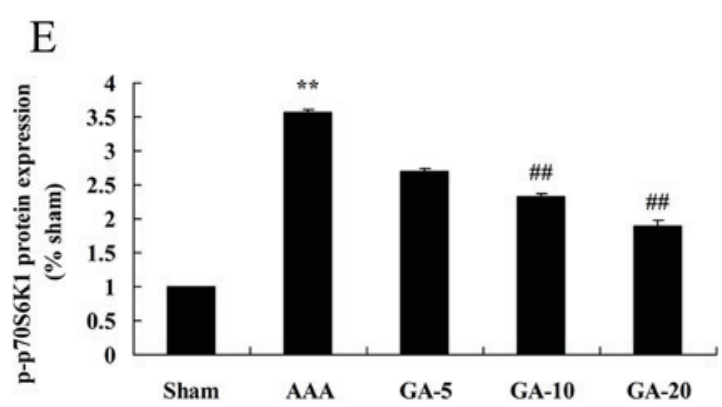

Figure 7. Effect of Gambogic acid on PI3K, p-Akt, p-mTOR, and p-p70S6K1 protein expression in AAA mice. (A) Representative western blot images. Quantification of (B) PI3K, (C) p-Akt, (D) p-mTOR and (E) p-p70S6K1 protein expression levels. ${ }^{* *} \mathrm{P}<0.01$ vs. sham group; ${ }^{\# \#} \mathrm{P}<0.01 \mathrm{vs}$. AAA model group. Sham, sham group; AAA, AAA model group; GA-5, Gambogic acid treatment group (5 mg/kg); GA-10, Gambogic acid treatment group (10 mg/kg); GA-20, Gambogic acid treatment group (20 mg/kg); AAA, abdominal aortic aneurysm; p-, phosphorylated; mTOR, mechanistic target of rapamycin; Akt, protein kinase B, PI3K, phosphatidylinositol 3-kinase.

Normally, NF- $\mathrm{kB}$ exists in the cytoplasm and binds to inhibitive protein I $\kappa \mathrm{B}$ to stay in an inactive state (34). When cells are stimulated, I $\mathrm{B}$ kinase complex (IKK) will be activated and phosphorylate $\mathrm{I} \kappa \mathrm{B}$ to dissociate $\mathrm{I} \kappa \mathrm{B}$ with NF- $\kappa \mathrm{B}$, then free NF- $\kappa$ B will quickly transfer into nucleus and bind to the target gene sequence specifically, thus regulating gene expression associated with various major pathophysiological reactions such as proliferation, differentiation, metastasis and apoptosis of cells, including the secretion of extracellular matrix degrading enzymes such as MMP and urokinase (35). In our experiments, Gambogic acid significantly suppressed NF- $\kappa \mathrm{B}$ protein expression in AAA mice. Liu et al (36) reported that GA induced apoptosis via suppression of NF- $\mathrm{KB}$ pathway of esophageal squamous cell carcinoma cells.

In conclusion, the results of the present study indicated that Gambogic acid prevents AngII-induced AAA incidence rate, edge leading aortic diameter and aortic wall thickness in AAA mice. These data support that Gambogic acid decreased the levels of proinflammatory cytokines, oxidative stress, and TGF- $\beta$, MMP-2, MMP-9 protein expression in AAA mice through the PI3K/Akt/mTOR and NF-kB signaling pathways. Furthermore, Gambogic acid treatment may provide a promising approach for the prevention of AAA in the future.

\section{Acknowledgements}

The authors would like to thank Dr Wang Qingshan for his help writing the manuscript.

\section{Funding}

No funding was received.

\section{Availability of data and materials}

The analyzed data sets generated during the study are available from the corresponding author on reasonable request.

\section{Authors' contributions}

PS designed the experiment; QL, PS and HL performed the experiment. PS analyzed the data.

\section{Ethics approval and consent to participate}

The present study was approved by the Animal Ethical and Welfare Committee of The First Hospital of Qiqihar City (Qiqihar, China). 


\section{Consent for publication}

Not applicable.

\section{Competing interests}

The authors declare they have no competing interests.

\section{References}

1. Zhang Y, Zhang D, Wang F, Xu D, Guo Y and Cui W: Serum miRNAs panel (miR-16-2*, miR-195, miR-2861, miR-497) as novel non-invasive biomarkers for detection of cervical cancer. Sci Rep 5: 17942, 2015.

2. Peng S, Gao D, Gao C, Wei P, Niu M and Shuai C: MicroRNAs regulate signaling pathways in osteogenic differentiation of mesenchymal stem cells (review). Mol Med Rep 14: 623-629, 2016.

3. Wang M, Liang L, Li L, Han K, Li Q, Peng Y, Peng X and Zeng K: Increased miR-424-5p expression in peripheral blood mononuclear cells from patients with pemphigus. Mol Med Rep 15: 3479-3484, 2017.

4. Svoboda M, Riha J, Wlcek K, Jaeger W and Thalhammer T: Organic anion transporting polypeptides (OATPs): Regulation of expression and function. Curr Drug Metab 12: 139-153, 2011.

5. Meyer Zu Schwabedissen HE, Böttcher K, Chaudhry A, Kroemer HK, Schuetz EG and Kim RB: Liver X receptor a and farnesoid $\mathrm{X}$ receptor are major transcriptional regulators of OATP1B1. Hepatology 52: 1797-1807, 2010.

6. Liao R, Yan F, Zeng Z, Farhan M, Little P, Quirion R, Srivastava LK and Zheng W: Amiodarone-induced retinal neuronal cell apoptosis attenuated by IGF-1 via counter regulation of the PI3k/Akt/FoxO3a pathway. Mol Neurobiol 54: 6931-6943, 2017.

7. Zhu W, Bijur GN, Styles NA and Li X: Regulation of FOXO3a by brain-derived neurotrophic factor in differentiated human SH-SY5Y neuroblastoma cells. Brain Res Mol Brain Res 126: 45-56, 2004.

8. Liu MH, Yuan C, He J, Tan TP, Wu SJ, Fu HY, Liu J, Yu S, Chen YD, Le QF, et al: Resveratrol protects PC12 cells from high glucose-induced neurotoxicity via PI3K/Akt/FoxO3a pathway. Cell Mol Neurobiol 35: 513-522, 2015.

9. Yoo HI, Kim BK and Yoon SK: MicroRNA-330-5p negatively regulates ITGA5 expression in human colorectal cancer. Oncol Rep 36: 3023-3029, 2016

10. Tian Y, Guo S, Wu X, Ma L and Zhao X: Minocycline alleviates sevoflurane-induced cognitive impairment in aged rats. Cell Mol Neurobiol 35: 585-594, 2015.

11. Yang ZJ, Wang YW, Li CL, Ma LQ and Zhao X: Pre-treatment with a Xingnaojing preparation ameliorates sevoflurane-induced neuroapoptosis in the infant rat striatum. Mol Med Rep 11: 1615-1622, 2015.

12. Chmielarz P, Konovalova J, Najam SS, Alter H, Piepponen TP, Erfle H, Sonntag KC, Schütz G, Vinnikov IA and Domanskyi A: Dicer and microRNAs protect adult dopamine neurons. Cell Death Dis 8: e2813, 2017.

13. Zeng Z, Wang X, Bhardwaj SK, Zhou X, Little PJ, Quirion R, Srivastava LK and Zheng W: The atypical antipsychotic agent, clozapine, protects against corticosterone-induced death of $\mathrm{PC} 12$ cells by regulating the $\mathrm{Akt} / \mathrm{FoxO} 3 \mathrm{a}$ signaling pathway. Mol Neurobiol 54: 3395-3406, 2017.

14. Kim HY, Kwon HY, Ha Thi HT, Lee HJ, Kim GI, Hahm KB and Hong S: MicroRNA-132 and microRNA-223 control positive feedback circuit by regulating FOXO3a in inflammatory bowel disease. J Gastroenterol Hepatol 31: 1727-1735, 2016.

15. Lian R, Lu B, Jiao L, Li S, Wang H, Miao W and Yu W: MiR-132 plays an oncogenic role in laryngeal squamous cell carcinoma by targeting FOXO1 and activating the PI3K/AKT pathway. Eur J Pharmacol 792: 1-6, 2016.

16. Breitkopf K, Nagy LE, Beier JI, Mueller S, Weng H and Dooley S: Current experimental perspectives on the clinical progression of alcoholic liver disease. Alcohol Clin Exp Res 33 $1647-1655,2009$.

17. Zubillaga-Guerrero MI, Alarcón-Romero Ldel C, Illades-Aguiar B, Flores-Alfaro E, Bermúdez-Morales VH, Deas $J$ and Peralta-Zaragoza O: MicroRNA miR-16-1 regulates CCNE1 (cyclin E1) gene expression in human cervical cancer cells. Int J Clin Exp Med 8: 15999-16006, 2015.
18. Ziaei S and Halaby R: Immunosuppressive, anti-inflammatory and anti-cancer properties of triptolide: A mini review. Avicenna J Phytomed 6: 149-164, 2016.

19. Wang F, Yin J, Lu Z, Zhang G, Li J, Xing T, Zhuang S and Wang N: Limb ischemic preconditioning protects against contrast-induced nephropathy via renalase. EBioMedicine 9: 356-365, 2016.

20. Liu SJ, Yin CX, Ding MC, Xia SY, Shen QM and Wu JD Berberine suppresses in vitro migration of human aortic smooth muscle cells through the inhibitions of MMP-2/9, u-PA, AP-1, and NF-кB. BMB Rep 47: 388-392, 2014.

21. Wang F, Zhang G, Lu Z, Geurts AM, Usa K, Jacob HJ, Cowley AW, Wang N and Liang M: Antithrombin III/SerpinC1 insufficiency exacerbates renal ischemia/reperfusion injury. Kidney Int 88: 796-803, 2015.

22. Yang X, Li CJ, Wan Y, Smith P, Shang G and Cui Q: Antioxidative fullerol promotes osteogenesis of human adipose-derived stem cells. Int J Nanomedicine 9: 4023-4031, 2014.

23. Wen J, Pei H, Wang X, et al: Gambogic acid exhibits anti-psoriatic efficacy through inhibition of angiogenesis and inflammation. J Dermatol Sci 74: 242-250, 2014.

24. Tian Y, Wu X, Guo S, Ma L, Huang W and Zhao X: Minocycline attenuates sevoflurane-induced cell injury via activation of $\mathrm{Nrf} 2$. Int J Mol Med 39: 869-878, 2017.

25. Zhou ZB, Yang XY, Tang Y, Zhou X, Zhou LH and Feng X: Subclinical concentrations of sevoflurane reduce oxidative stress but do not prevent hippocampal apoptosis. Mol Med Rep 14: 721-727, 2016

26. Qu Y, Zhang G, Ji Y, Zhua H, Lv C and Jiang W: Protective role of gambogic acid in experimental pulmonary fibrosis in vitro and in vivo. Phytomedicine 23: 350-358, 2016.

27. Zhu G, Wang X, Wu S and Li Q: Involvement of activation of PI3K/Akt pathway in the protective effects of puerarin against MPP+-induced human neuroblastoma SH-SY5Y cell death. Neurochem Int 60: 400-408, 2012.

28. Xiang J, Pan J, Chen F, Zheng L, Chen Y, Zhang S and Feng W: L-3-n-butylphthalide improves cognitive impairment of APP/PS1 mice by BDNF/TrkB/PI3K/AKT pathway. Int J Clin Exp Med 7: 1706-1713, 2014.

29. Hossini AM, Quast AS, Plötz M, Grauel K, Exner T, Küchler J, Stachelscheid H, Eberle J, Rabien A, Makrantonaki E and Zouboulis CC: PI3K/AKT signaling pathway is essential for survival of induced pluripotent stem cells. PLoS One 11: e0154770, 2016.

30. Qi Q, Gu H, Yang Y, et al: Involvement of matrix metalloproteinase 2 and 9 in gambogic acid induced suppression of MDA-MB-435 human breast carcinoma cell lung metastasis. J Mol Med (Berl) 86: 1367-1377, 2008.

31. Luo T, Liu G, Ma H, Lu B, Xu H, Wang Y, Wu J, Ge P and Liang J: Inhibition of autophagy via activation of PI3K/Akt pathway contributes to the protection of ginsenoside $\mathrm{Rbl}$ against neuronal death caused by ischemic insults. Int J Mol Sci 15: 15426-15442, 2014.

32. Meng Y, Wang W, Kang J, Wang $X$ and Sun L: Role of the $\mathrm{PI} 3 \mathrm{~K} / \mathrm{AKT}$ signalling pathway in apoptotic cell death in the cerebral cortex of streptozotocin-induced diabetic rats. Exp Ther Med 13: 2417-2422, 2017.

33. Wang F, Zhang W, Guo L, Bao W, Jin N, Liu R, Liu P, Wang Y, Guo Q and Chen B: Gambogic acid suppresses hypoxia-induced hypoxia-inducible factor- $1 \alpha /$ vascular endothelial growth factor expression via inhibiting phosphatidylinositol 3-kinase/Akt/mammalian target protein of rapamycin pathway in multiple myeloma cells. Cancer Sci 105: 1063-1070, 2014

34. Yufune S, Satoh Y, Akai R, Yoshinaga Y, Kobayashi Y, Endo S and Kazama T: Suppression of ERK phosphorylation through oxidative stress is involved in the mechanism underlying sevoflurane-induced toxicity in the developing brain. Sci Rep 6: 21859, 2016.

35. Guo XQ, Cao YL, Hao F, Yan ZR, Wang ML and Liu XW: Tangeretin alters neuronal apoptosis and ameliorates the severity of seizures in experimental epilepsy-induced rats by modulating apoptotic protein expressions, regulating matrix metalloproteinases, and activating the PI3K/Akt cell survival pathway. Adv Med Sci 62: 246-253, 2017.

36. Liu WY, Wu XU, Liao CQ, Shen J and Li J: Apoptotic effect of gambogic acid in esophageal squamous cell carcinoma cells via suppression of the NF-kappaB pathway. Oncol Lett 11: $3681-3685,2016$ 